\title{
Gender difference in adrenal developmental toxicity induced by dexamethasone and its intrauterine programming mechanism
}

\author{
Yawen Chen ${ }^{1}$, Dan $\mathrm{Xu}^{2}$, Xuan Xia ${ }^{2}$, Guanghui Chen ${ }^{3}$, Jiangang $\mathrm{Cao}^{2}$, Hao Xiao ${ }^{4}$, Liaobin \\ $\mathrm{Chen}^{4}$, and Hui Wang ${ }^{1}$ \\ ${ }^{1}$ Wuhan University School of Basic Medical Sciences \\ ${ }^{2}$ Wuhan University \\ ${ }^{3}$ School of Basic Medical Science of Wuhan University \\ ${ }^{4}$ Zhongnan Hospital of Wuhan University
}

March 31, 2021

\begin{abstract}
Background and Purpose: Dexamethasone is widely used in preterm labor and related diseases. However, prenatal dexamethasone exposure (PDE) can cause multi-organ developmental toxicities in offspring. Our previous study found the occurrence of fetal-originated diseases were associated with adrenal developmental programming alteration in offspring. Here, we investigated the effects of PDE on the adrenal function in offspring and its intrauterine programming mechanism. Experimental Approach: A rat model of PDE was established to observe the alteration of adrenal steroidogenesis in offspring. Further, we confirmed the gender difference of adrenal steroidogenesis and its molecular mechanism combined with in vivo and in vitro experiment. Key Results: PDE caused a decrease in adrenal steroidogenic function in fetal rats, but decreased in males and increased in females after birth. Meanwhile, the adrenal H3K14ac level and expression of 11ß-hydroxysteroid dehydrogenase 2 (11ß-HSD2) in PDE offspring were decreased in males and increased in females, suggesting 11ß-HSD2 might mediate gender difference of adrenal function. We further confirmed dexamethasone inhibited the H3K14ac level and expression of 11ß-HSD2 through GR/SP1/p300 pathway. After bilateral testectomy or ovariectomy in adult PDE offspring rats, adrenal 11 $\beta$-HSD2 expression and steroidogenic function were both reduced. Using rat primary fetal adrenal cells, the differential expression in AR and ER $\beta$ were proved to involve in regulating the gender difference of $11 \beta-H S D 2$ expression. Conclusion and Implications: This study demonstrated the gender difference in adrenal steroidogenic function of PDE offspring after birth, and elucidates a sex hormone receptor-dependent epigenetically regulating mechanism for adrenal 11 $\beta$-HSD2 programming alteration.
\end{abstract}

Gender difference in adrenal developmental toxicity induced by dexamethasone and its intrauterine programming mechanism

Yawen Chen ${ }^{1, \#}$, Dan Xu ${ }^{1,3, \#}$, Xuan Xia ${ }^{1}$, Guanghui Chen ${ }^{3}$, Hao Xiao ${ }^{2,3}$, Liaobin Chen ${ }^{2,3,}{ }^{* *}$, Hui Wang ${ }^{1,3, *}$

${ }^{1}$ Department of Pharmacology, Wuhan University School of Basic Medical Sciences, Wuhan 430071, China;

2 Department of Orthopedic Surgery, Zhongnan Hospital of Wuhan University, Wuhan 430071, China;

${ }^{3}$ Hubei Provincial Key Laboratory of Developmentally Originated Disease, Wuhan 430071, China.

\# Yawen Chen and Dan Xu contributed equally to this work.

Corresponding authors

* Hui Wang, M.D., Ph.D., Department of Pharmacology, Wuhan University School of Basic Medical Sciences. Tel: +86-13627232557. E-mail: wanghui19@whu.edu.cn. 
** Liaobin Chen, Ph.D., Department of Orthopedic Surgery, Zhongnan Hospital of Wuhan University. Tel: +86-13618610516. E-mail: lbchen@whu.edu.cn.

\section{Acknowledgments}

This work was supported by grants from the National Natural Science Foundation of China (No. 81673524, 82030111, 81973405), the National Key Research and Development Program of China (No. 2020YFA0803900), the Major Technological Innovation Projects of Hubei Province (No. 2019ACA140, 2020BCA071), and Medical Science Advancement Program (Basic Medical Sciences) of Wuhan University (No. TFJC2018001).

\section{Conflict of interest disclosure}

The authors declare no conflicts of interest.

\section{Author contribution statement}

H. Wang conceived the study; H. Wang, Y. Chen and D. Xu participated in the study design. Y. Chen, X. Xia, and G. Chen performed the experiments and acquired the data. Y. Chen and D. Xu wrote the manuscript; H. Wang, L. Chen, and H. Xiao revised the manuscript; All authors read and approved the final manuscript.

\section{Data availability statement}

The data that support the findings of this study are available from the corresponding author upon reasonable request. Some data may not be made available because of privacy or ethical restrictions.

\section{Bullet point summary}

\section{What is already known}

Prenatal dexamethasone exposure can cause multi-organ developmental toxicities and increase susceptibility to metabolic diseases in offspring and exist gender difference, which is related to glucocorticoid level in body.

\section{What this study adds}

The gender difference in metabolic diseases induced by prenatal dexamethasone exposure can be explained from the perspective of the intrauterine origin of adrenal development.

A sex hormone receptor-dependent epigenetically regulating mechanism for adrenal 11 $\beta$-HSD2 programming alteration mediated the abnormal adrenal steroidogenic function induced by prenatal dexamethasone exposure in adult offspring.

\section{What is the clinical significance}

$11 \beta-H S D 2$ could be an early warning targets for prenatal dexamethasone exposure-induced abnormal adrenal development and metabolic diseases in adult offspring.

\section{Abstract}

Background and Purpose:Dexamethasone is widely used in preterm labor and related diseases. However, prenatal dexamethasone exposure (PDE) can cause multi-organ developmental toxicities in offspring. Our previous study found the occurrence of fetal-originated diseases were associated with adrenal developmental programming alteration in offspring. Here, we investigated the effects of PDE on the adrenal function in offspring and its intrauterine programming mechanism.

Experimental Approach: A rat model of PDE was established to observe the alteration of adrenal steroidogenesis in offspring. Further, we confirmed the gender difference of adrenal steroidogenesis and its molecular mechanism combined with in vivo and in vitroexperiment. 
Key Results: PDE caused a decrease in adrenal steroidogenic function in fetal rats, but decreased in males and increased in females after birth. Meanwhile, the adrenal H3K14ac level and expression of 11 $\beta$ hydroxysteroid dehydrogenase 2 (11/-HSD2) in PDE offspring were decreased in males and increased in females, suggesting 11/-HSD2 might mediate gender difference of adrenal function. We further confirmed dexamethasone inhibited the H3K14ac level and expression of 11/-HSD2 through GR/SP1/p300 pathway. After bilateral testectomy or ovariectomy in adult PDE offspring rats, adrenal 11ß-HSD2 expression and steroidogenic function were both reduced. Using rat primary fetal adrenal cells, the differential expression in $\mathrm{AR}$ and $\mathrm{ER} \beta$ were proved to involve in regulating the gender difference of $11 \beta$-HSD2 expression.

Conclusion and Implications:This study demonstrated the gender difference in adrenal steroidogenic function of PDE offspring after birth, and elucidates a sex hormone receptor-dependent epigenetically regulating mechanism for adrenal 11 $\beta$-HSD2 programming alteration.

Keywords : Prenatal dexamethasone exposure; adrenal function; sex hormone receptors; gender differences; $11 \beta$-hydroxysteroid dehydrogenase 2

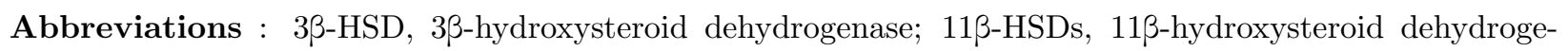
nases; AR, androgen receptor; CORT, corticosterone; ER $\beta$, estrogen receptor beta; GD, gestation day; GR, glucocorticoid receptor; H3K14ac, histone 3 lysine 14 acetylation; IUGR, intrauterine growth retardation; NR4A1, nuclear receptor subfamily 4 group A member 1; OVX, ovariectomy; P450c11, steroid 11 $\beta$ hydroxylase; P450c21, steroid 21-hydroxylase; P450scc, cytochrome P450 cholesterol side-chain cleavage; PDE, prenatal dexamethasone exposure; PW, postnatal week; SP1, special protein 1; StAR, steroidogenic acute regulatory protein; TDX, testectomy.

\section{Introduction}

Dexamethasone, as a long-acting synthetic glucocorticoid with highly fat-soluble, is easy to penetrate the placenta. It can promote fetal lung maturation, thereby reducing the incidence of neonatal respiratory distress syndrome and perinatal mortality. Therefore, it is widely used in preterm births and related pregnancy diseases (Roberts et al. , 2017). Based on the maternal and child health survey data from 359 institutions in 29 countries, the World Health Organization reported that the prevalence of prenatal synthetic glucocorticoid treatment for premature labor is $54 \%$, and reaches $91 \%$ in some countries, and $80 \%$ of premature infants received preventive therapy in Asia. Although the efficacy of prenatal dexamethasone in the treatment of preterm labor is positive, accumulated epidemiological investigations and clinical studies have found that dexamethasone has a "double-edged sword" effect. Several clinical and animal studies have demonstrated that prenatal dexamethasone exposure (PDE) was associated with fetal growth restriction and the occurrence of many chronic diseases, such as hypertension and neuropsychiatric disorders (Trautmanet al. , 1995; Doyle et al. , 2000)(Long et al. , 2012; Xu et al. , 2018). Therefore, the safety of dexamethasone application during pregnancy has become a hot topic in the global medical community.

There are significant gender differences in the epidemiology, clinical manifestation, pathophysiology, treatment, and outcomes of various metabolic diseases, such as non-alcoholic fatty liver, cardiovascular and cerebrovascular diseases (Ayonrinde et al. , 2011; Ojeda et al. , 2014; Dasinger et al. , 2016). The same environmental exposure during pregnancy has different effects on the occurrence time, course and outcome of chronic diseases in male and female offspring (van Abeelen et al. , 2011; Schaafsma et al. , 2017). Although these diseases only show clear disease states in adulthood or old age, the roots can often be traced back to childhood, infancy, and even fetal period (Millershipet al. , 2019). The hypothalamus-pituitary-adrenal (HPA) axis is an essential neuroendocrine axis of the body. The adrenal gland is the earliest and fastest development part of the HPA axis, and its normal development is necessary to maintain the metabolic homeostasis (Moisiadis et al. , 2014a). It has been found that glucocorticoids secreted by adrenal gland are an important inducement for the occurrence and development of the fetal-originated metabolic diseases. Adverse environments during pregnancy can cause adrenal dysfunction in offspring (Waddell et al. , 2010; Pawluski et al. , 2012), and even gender differences (Pinheiroet al. , 2011), while the difference in adrenal function also increases gender differences in metabolic diseases (Arnetzet al. , 2015). Therefore, the gender differences 
in adrenal dysfunction are involved in the occurrence of gender differences in the fetal-originated metabolic diseases. Studies have shown that PDE-induced developmental restrictions in embryos and neonates are more evident in male offspring (Tomaszewska et al. , 2012; O'Connell et al. , 2013; Yun et al. , 2016), while the female offspring were more vulnerable to adult metabolic diseases than male offspring (Carpenter et al. , 2017). However, it has not been reported whether there are gender differences in adrenal function in PDE offspring as well as its underlying mechanism.

Glucocorticoids generally exhibit their biological effects through glucocorticoid receptor (GR), which is affected by both circulating glucocorticoid levels and regulated by glucocorticoid-metabolic enzyme system, $11 \beta$-hydroxysteroid dehydrogenases (11ß-HSDs) (McNeil et al. , 2007). As a critical fetal developmentrelated gene, $11 \beta$-HSD2 is a high-affinity $\mathrm{NAD}(\mathrm{H})$-dependent dehydrogenase, which is mainly distributed in the placenta, kidney, adrenal gland, colon, etc. 11ß-HSD2 can catalyze active glucocorticoids (cortisol in humans, corticosterone in rodents) into their inactive metabolites (cortisone, 11-dehydrocorticosterone), thus maintaining an appropriate concentration range of tissue and intracellular glucocorticoids (Chapman et al. , 2013). It has been reported that 11ß-HSD2 is involved in the occurrence of metabolic diseases by regulating glucocorticoid levels (Bailey, 2017). A study has shown when glucocorticoid levels rise due to the imbalance of the 11ß-HSDs system, the phenotypes of metabolic syndrome such as obesity, insulin resistance, dyslipidemia and hypertension occur (Masuzakiet al. , 2001). Therefore, we speculated that 11ß-HSD2 is involved in regulating adrenal developmental programming and metabolic homeostasis through inactivating endogenous glucocorticoids, thereby changing the circulating glucocorticoid levels of PDE offspring after birth, leading to their increased susceptibility to metabolic syndrome and related diseases.

In this study, we aim to confirm the gender difference in adrenal steroidogenic function of PDE offspring rats and further analyze the correlation between adrenal local 11ß-HSD2 expression and steroidogenic function. Moreover, we propose an androgen receptor (AR)/ estrogen receptor beta (ER $\beta$ )-associated intrauterine epigenetic programming mechanism that leads to gender difference in 11ß-HSD2 expression of male and female PDE offspring. This study illuminates the gender differences in the PDE-induced adrenal steroidogenic function from the perspective of the intrauterine origin of adrenal development, providing experimental evidence and novel ideas for exploring early prevention and control strategies of fetal-originated adult diseases.

\section{Materials and methods}

\subsection{Animals and treatment}

Specific pathogen-free Wistar rats (certification No. 42000600002258, license No. SCXK (E) 20170018) weighing $280 \pm 20 \mathrm{~g}$ (males) and $200 \pm 20 \mathrm{~g}$ (females) were purchased from the Experimental Center of the Hubei Medical Scientific Academy (Wuhan, China). Animal experiments were handled in the Center for Animal Experiments of Wuhan University (Wuhan, China), which is accredited by the Association for the Assessment and Accreditation of Laboratory Animal Care International (AAALAC International). The protocol was approved by the Committee on the Ethics of Animal Experiments of the Wuhan University School of Medicine (permit number: JC2020-015). All experimental animal procedures were performed based on the Chinese Animal Welfare Committee.

The rats were housed in metal cages with wire-mesh floors in an air-conditioned room under standard conditions (room temperature: 18-22degC; humidity: 40\%-60\%; light cycle: 12 -hour light-dark cycle; 10-15 air changes per hour) and allowed free access to rat chow and tap water. All rats were acclimated one week before experimentation, and two female rats and one male rat were placed together in a cage for mating overnight. The sperm detected on vaginal smear confirmed the mating, and the mating date was gestation day (GD) 0. Pregnant rats were randomly divided into either the control group or the PDE group. The pregnant rats were subcutaneously injected with $0.2 \mathrm{mg} / \mathrm{kg}^{*} \mathrm{~d}$ dexamethasone (Shuanghe, Pharmaceutical Co., Wuhan, China) or saline of equal volume every morning (between 08:00-09:00 a.m.) from GD9 to GD20.

A part of pregnant rats was anesthetized with $2 \%$ isoflurane and euthanized on GD20 ( $\mathrm{n}=12$ per group), and the blood sample was collected from the carotid artery. Those dams with litter sizes of 8 to 14 were considered qualified. The pups were weight, and the average weight of each litter was calculated, which was 
thought as a single $\mathrm{n}$ value. Intrauterine growth retardation (IUGR) was diagnosed when the body weight of a fetus was 2 standard deviation below the mean body weight of fetuses in the control group. The fetal rats were sacrificed for collecting blood and adrenals. Fetal blood samples were collected and serum was separated. For morphological purposes, the right adrenals of five fetal rats in each group were randomly selected for $24 \mathrm{~h}$ in $4 \%$ paraformaldehyde, then dehydrated in ethanol and embedded in paraffin. The remaining adrenals were immediately frozen in liquid nitrogen and stored at -80degC for further analysis.

The other pregnant rats $(\mathrm{n}=12$ per group) delivered normally. The pregnant rats with litter size (8-14) were considered qualified, therefore eight litters of both control and PDE groups were retained after birth. The number of fetal rats was normalized to 10 for each litter to ensure balanced lactation nutrition; the male/female ratio was approximately 1:1. The pups were raised normally until weaning at postnatal week (PW) 4. After weaning, three male and three female pups were randomly selected from each pregnant rat in both the control and PDE groups, and they were processed in three batches ( $\mathrm{n}=8$ per group). The first and the second batches were euthanized with $2 \%$ isoflurane at PW12 and PW28, respectively. The third batch underwent bilateral testectomy (TDX) and ovariectomy (OVX) (including the control and PDE groups) on PW22 and were killed at PW28. Then, the blood samples of three batch from the carotid artery were collected, the serum and adrenal tissues were separated and immediately frozen in liquid nitrogen, followed by storage at -80 for subsequent analyses. The right adrenals of five rats in each group were randomly selected and processed for immunohistochemistry analysis.

\subsection{Corticosterone, testosterone, estradiol and cortisol concentration analysis}

We combined serum samples of 3 fetal rats from the same litter into one sample, so the serum samples from 8 litters in each group constituted $n=8$ /group. Since the combined fetal rats' samples originally came from the same litter, their genetic backgrounds were identical, so the confounding factor was not apparent. The concentration of serum corticosterone was measured by the ELISA assay kit (R\&D Systems, Inc., Minneapolis, MN, USA) according to the manufacturer's protocol. The limit of detection for corticosterone concentration was $0.39 \mathrm{ng} / \mathrm{mL}$. The intra-assay and inter-assay coefficients of variation for corticosterone were 5.0 and $7.2 \%$, respectively. The concentration of serum testosterone was measured using a testosterone ELISA kit (R\&D Systems, Inc., Minneapolis, MN, USA) according to the manufacturer's protocol. The intra- and inter-assay coefficients of variation were $10.0 \%$ and $15.0 \%$ for testosterone, respectively. The concentration of serum estradiol was measured by ELISA assay kit (R\&D Systems, Inc., Minneapolis, MN, USA) according to the manufacturer's protocol. The intra- and inter-assay coefficients of variation were $6.0 \%$ and $7.1 \%$ for estradiol, respectively.

The content of cortisol in cell supernatant was measured by the ELISA assay kit (R\&D Systems, Inc., Minneapolis, MN, USA) according to the manufacturer's protocol ( $\mathrm{n}=6$ per group). The limit of detection for cortisol concentration was $0.111 \mathrm{ng} / \mathrm{mL}$. The intra-assay and inter-assay coefficients of variation for corticosterone were 6.3 and $10.4 \%$, respectively.

\subsection{Immunohistochemistry analysis}

For immunohistochemistry quantification, the right adrenals of 5 rats in each group were selected. We fixed adrenals in $4 \%$ paraformaldehyde for $24 \mathrm{~h}$, embedded with paraffin. Samples were cut into $5 \mu \mathrm{m}-$ thick slices along the longitudinal axis. For immunohistochemical staining, the slices were dewaxed and washed in PBS. After antigen retrieval, the hydrated sections were then incubated in $3 \% \mathrm{H}_{2} \mathrm{O}_{2}$ for 15 min to quench the endogenous peroxidase activity. Sections were then blocked in 3\% BSA (Servicebio, Wuhan, China) at room temperature for $1 \mathrm{~h}$ and incubated with a primary antibody of 11ß-HSD2 (1:1000 dilution, sc-365529, Santa Cruz, CA, USA), or NR4A1 (1:1000 dilution, abs124596, Absin, Shanghai, China), or nuclear receptor subfamily 4 group A member 1 (NR4A1), or steroidogenic acute regulatory protein (StAR) (1:1000 dilution, A16432, ABclonal, Wuhan, China) at $4^{\circ} \mathrm{C}$ overnight. After washing with PBS, the sections were incubated with a biotinylated secondary antibody (anti-mouse or anti-rabbit conjugated with horseradish peroxidase, 1:100 dilution, AS014 and AS003, ABclonal, Wuhan, China) for $1 \mathrm{~h}$ and then with an avidin-biotinylated horseradish peroxidase complex solution according to the manufacturer's directions. 
Finally, peroxidase activity was determined with a DAB staining kit (Gene Tech Company, Ltd., Shanghai, China). For the quantitative analysis of $11 \beta-H S D 2$, NR4A1 and StAR, Image-Pro Plus software (version 6.1, Media Cybernetics, Silver Spring, USA) was used to count and analyze the number of positive cells in each field, and the average values of the five fields were calculated to obtain the data of each sample. The immunohistochemistry results of $11 \beta$-HSD2, NR4A1 and StAR were statistically analyzed by $\mathrm{n}=5$ per group to ensure the accuracy of sample size and quantification.

\subsection{Cell culture and treatment}

The human adrenocortical cell line (NCI-H295R) was purchased from the Shanghai Zishi Biotechnology Co., Ltd. (Shanghai, China). Cells were cultured in a $5 \% \mathrm{CO}_{2}$ humidified incubator at 37. The standard medium for NCI-H295R cells was DMEM supplemented with $10 \%$ fetal bovine serum, $1 \%$ streptomycin, and penicillin. The medium was changed every other day, and the cells were split at a ratio of 1:3 with trypsin after reaching confluence. The cells were treated with various concentrations of dexamethasone $(0,20,100$, $500 \mathrm{nM}$ ) for $24 \mathrm{~h}$ and then harvested for further analysis. Furthermore, prior to transfection, the cells were seeded in six-well plates at a density of $4 \times 10^{5}$ cells per well. After $24 \mathrm{~h}$, the cells were treated with 50 nM GR siRNA oligo, or $50 \mathrm{nM}$ SP1 plasmid, or $50 \mathrm{nM}$ 11ß-HSD2 plasmid (Gene Pharma Co., Jiangsu, China) transfection using Lipofectamine 3000 according to the manufacturer's protocol, or treated with 30 nM CTPB (APExBio, Houston, USA). And $8 \mathrm{~h}$ later, the cells were replaced with a full-medium containing $500 \mathrm{nM}$ dexamethasone. The cells were harvested after $24 \mathrm{~h}$ for further analysis.

Adrenal primary cells were isolated from fetal male and female Wistar rats at GD20. Briefly, the adrenals of rats were dissected under an anatomy scope. Minced adrenal was incubated in $0.025 \%$ trypsin at $37^{\circ} \mathrm{C}$ for $10 \mathrm{~min}$. The cells liberated during digestion were collected, cultured and plated at a density of $4 \times 10^{5}$ cells per well in 6-well plates in culture medium (DMEM medium with $10 \%$ fetal bovine serum, $1 \%$ streptomycin and penicillin). To qualitatively assessed the rat adrenal cells, we adopted the immunofluorescence method to detect the expression of cytochrome P450 Family 11 Subfamily B Member 1 (CYP11B1) and CYP11B2 in our previous study (Chen et al. , 2018a). The cells were treated with $500 \mathrm{nM}$ dexamethasone for $24 \mathrm{~h}$ and then harvested for further analysis. Another batch of cells were treated with $10 \mathrm{nM} \mathrm{DHT}$, or $50 \mathrm{nM}$ ER $\beta$ siRNA transfection using Lipofectamine 3000 according to the manufacturer's protocol. After $8 \mathrm{~h}$, the cells were replaced with a full-medium containing $500 \mathrm{nM}$ dexamethasone. The cells were harvested after 24 $\mathrm{h}$ for further analysis.

\subsection{Total RNA extraction, reverse transcription and real-time quantitative PCR (RT-qPCR)}

We combined the adrenal tissue of 3 fetal rats from the same litter into one sample, and each group of 8 fetal adrenal samples constituted $n=8 /$ group. The adrenals of adult offspring were not combined due to their sufficient size. In the cell experiment, RNA was extracted by homogenizing each cell pore plate and was thought as a single $\mathrm{n}$ value ( $\mathrm{n}=6$ per group). Total RNA was extracted according to the manufacturer's protocol. The concentration and purity of the isolated total RNA were determined by spectrophotometer (NanoDrop 2000C, Thermo), and the total RNA concentration was adjusted to $1 \mu \mathrm{g} / \mu \mathrm{L}$. To convert the total RNA into cDNA, we prepared single-strand cDNA from $2 \mu \mathrm{g}$ of total RNA according to the protocol of the reverse transcription reagent kit (Takara Biotechnology Co., Dalian, Liaoning, China). Then, RTqPCR was performed using an SYBR Green qPCR Master Mix kit and ABI Step One RT-qPCR thermal cycle apparatus (ABI Step one, USA) with 40 cycles. The cycle threshold (Ct) was detected, and the relative expression of genes was determined using the $2^{-} \Delta \Delta^{\text {a } T}$ method with normalization to glyceraldehyde3 -phosphate dehydrogenase (GAPDH) expression and used as a quantitative control. The sequences of primers used in this experiment are shown in Table S1. All primers were synthesized by Sangon Biotech Co., Ltd. (Shanghai, China).

\subsection{Total protein extract and Western blotting assay}

Protein was extracted from NCI-H295R cells ( $\mathrm{n}=5$ per group) using a RIPA buffer containing protease inhibitors (Complete Mini; Roche) and phosphatase inhibitors (04906845001, PhosSTOP; Roche). Protein concentrations was quantified using a BCA Protein Assay Kit (P0011; Beyotime). Cytoplasmic protein and 
nucleic protein were respectively extracted by the nuclear and cytoplasmic protein extraction kit following the manufacturer's protocols. Equal amounts of protein lysates (15 $\mu \mathrm{g}$ per lane) were resolved by SDS-PAGE on $10 \%$ polyacrylamide gels, transferred to polyvinylidene difluoride membranes (IPVH00010, Immobilon). Membranes were blocked with $5 \%$ nonfat dried milk for $1 \mathrm{~h}$, and then incubated with primary antibodies for GR (1:100 dilution, sc-376426, Santa Cruz, CA, USA), or SP1 (1:5000 dilution, ab13370, Abcam, Cambridge, UK), or p300 (1:1000 dilution, ab14984, Abcam, Cambridge, UK), or 11ß-HSD2 (1:1000 dilution, sc-365529, Santa Cruz, CA, USA), or NR4A1 (1:1000 dilution, abs124596, Absin, Shanghai, China), or StAR (1:1000 dilution, A16432, ABclonal, Wuhan, China), or AR (1:2000 dilution, ab133273, Abcam, Cambridge, UK), or ER $\beta$ (1:200 dilution, sc-53494, Santa Cruz, CA, USA) at $4^{\circ} \mathrm{C}$ overnight. After washing three times with TBST, the membranes were incubated with secondary antibodies (anti-mouse or anti-rabbit conjugated with horseradish peroxidase, 1:100 dilution, AS014 and AS003, ABclonal, CA, USA) for $1 \mathrm{~h}$. Finally, the membranes were detected using the ECL Plus Western Blotting Detection System (Applied Biosystems). Band intensity was quantified using Quantity One (Bio-Rad, Shanghai, China). To verify the relative amounts of protein in each lane, the level of GAPDH was determined as an internal control.

\subsection{Co-immunoprecipitation (Co-IP) assay}

Co-IP assay was performed to detect the interaction of GR with AR or ER $\beta$ in adrenal primary cells (n $=5$ per group). After washing with ice-cold PBS, cells were lysed in $1.2 \mathrm{~mL}$ lysis buffer at -80 for $30 \mathrm{~min}$. The samples were centrifugated with 14,000 rpm at 4 for $15 \mathrm{~min}$, and the supernatant was transferred to a new column. And then the samples were divided into three parts: the first was used for input protein, the other two were performed using $1 \mu \mathrm{g}$ of either a mock antibody IgG (1:100 dilution, A19711, ABclonal, Wuhan, China) as a control or GR antibody (1:100 dilution, sc-376426, Santa Cruz, CA, USA) respectively followed by incubation overnight at $4^{\circ} \mathrm{C}$ with gentle shaking. And then, the samples were incubated with protein $\mathrm{G}$ magnetic beads at $4^{\circ} \mathrm{C}$ for $6 \mathrm{~h}$. After immunoprecipitation, the samples were washed with lysis buffer. After three items of washing, retained proteins were mixed with $30 \mu \mathrm{L}$ loading buffer at 100 for 10 min. Protein complexes and input protein were then detected by Western blotting.

\subsection{Chromatin immunoprecipitation-polymerase chain reaction (ChIP-PCR)}

We combined the adrenal tissue of 3 fetal rats from the same litter into one sample, and each group of 6 fetal adrenal samples constituted $n=6$ /group. The adrenals of adult offspring were not combined due to their sufficient size. In the cell experiment, DNA was extracted by homogenizing each cell pore plate and was thought as a single $\mathrm{n}$ value $(\mathrm{n}=6$ per group). The homogenates of adrenal tissues or scraped cells were collected and fixed in $1 \%$ formaldehyde for chromatin cross-linking and added $125 \mathrm{mM}$ glycine to stop the reaction. The samples were then centrifuged and resuspended in $0.5 \mathrm{~mL}$ lysis buffer containing protease inhibitors. Cell lysates were sonicated to shear DNA to lengths of approximately 200 base pairs and transferred to a new tube with ChIP dilution buffer. Chromatin was incubated overnight at $4^{\circ} \mathrm{C}$ on nutator/rocker with specific antibody for H3K9ac (1:50 dilution, A7255, ABclonal, Wuhan, China), or H3K14ac (1:50 dilution, A7254, ABclonal, Wuhan, China), or H3K27ac (1:50 dilution, A7253, ABclonal, Wuhan, China) or goat anti-rabbit IgG (1:50 dilution, AC005, ABclonal, Wuhan, China). The immunoprecipitated DNA-protein complex with beads was collected by centrifugation and washed sequentially with low-salt, high-salt, $\mathrm{LiCl}$ immune complex, and Tris-EDTA washing buffer solutions. Freshly prepared elution buffer (1\% SDS, $0.1 \mathrm{M}$ $\mathrm{NaHCO}_{3}$ ) was used to elute the DNA protein complex. The samples were incubated at $4^{\circ} \mathrm{C}$ overnight on a rocker, followed by incubation with BSA treated Proteinase K (EO0491, Beyotime Biotechnology, Nanjing, China) at $65^{\circ} \mathrm{C}$ overnight. After elution and purification with a TIAN quick Midi Purification kit (Tiangen Biotech Co., Beijing, China) according to the manufacturer's instructions, the resultant DNA was used for RT-qPCR analysis. The primers are listed in Table S2.

\subsection{Statistical analysis}

SPSS 20.0 (SPSS Science Inc., Chicago, Illinois, USA) and Prism 6.0 (GraphPad Software, La Jolla, CA, USA) were used to analyze experimental data. The declared group size was the number of independent values, and statistical analysis was done using these independent values. All data were expressed as mean \pm S.E.M. 
The outliers were included in data analysis and presentation. To reduce unwanted sources of variation, some data were conducted data normalization, which means the correction of test values to baseline or control group values. Student's two-tailed $t$ test was performed on one factor of prenatal dexamethasone treatment. For the data from multigroup studies, one-way ANOVA (or equivalent) achieved the chosen necessary level of statistical significance and there was no significant variance inhomogeneity. The Pearson correlation analysis was used to analyze the correlation between two indicators. A value of $P<0.05$ was considered statistically significant.

\section{Results}

\subsection{Gender differencechanges in adrenal steroidogenic function of PDE offspring after birth}

Firstly, pregnant rats were injected subcutaneously with $0.2 \mathrm{mg} / \mathrm{kg} \cdot \mathrm{d}$ dexamethasone during GD9-20 to demonstrate the gender differences in serum corticosterone levels and adrenal steroidogenic function in offspring rats of different time on GD20, PW12 and PW28. We found that, compared with the respective control, the body weights were both significantly decreased in male and female fetal rats of the PDE group (Fig 1a), whereas the IUGR rates were markedly increased (Fig 1b). Meanwhile, the serum corticosterone concentration, and the mRNA expression levels of adrenal NR4A1 (a key transcriptional activator of steroidogenesis) and related steroidogenic genes, including StAR, P450scc, 33-HSD, and P450c11, were reduced (Fig 1c-e); the protein expression levels of adrenal NR4A1 and StAR by immunohistochemical analysis were also significantly decreased (Fig 1f, g).

However, at PW12 and PW28, serum corticosterone concentrations and mRNA expression levels of adrenal steroidogenic genes in the male PDE offspring were inhibited (Fig 1h, i, k and l), whereas in the females significantly increased (Fig $1 \mathrm{~h}, \mathrm{j}, \mathrm{k}, \mathrm{m}$ ). Meanwhile, the protein expression changes in adrenal NR4A1 and StAR were consistent with those of mRNA expression (Fig 1n, o). These results suggested that the adrenal steroidogenic function in the male and female PDE fetal rats were both inhibited but presented a significant gender difference after birth, showing a sustained decrease in the male offspring, whereas an increase in the females.

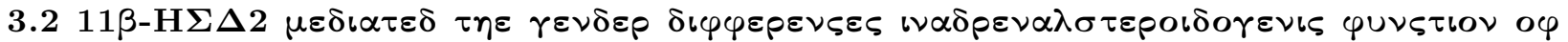

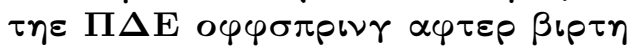

To explore whether 11 $\beta$-HSD2 is related to the gender difference in adrenal steroidogenic function, we examined the expression of adrenal 11ß-HSD2 in the PDE offspring rats before and after birth. Compared with the control, the mRNA expression of adrenal 11ß-HSD2 in the male PDE fetal rats was decreased to some degree while in the females significantly increased (Fig 2a). Immunohistochemical analysis also showed that the protein expression of $11 \beta-H S D 2$ in the male PDE fetal rats was markedly decreased while in the females significantly increased (Fig 2b). The above changes could continue to PW12 and PW28 (Fig 2c, d, g). It is suggested that the expression of adrenal 11ß-HSD2 in the PDE offspring shows significant gender difference, which extends from intrauterine to postnatal adulthood. Furthermore, we analyzed the correlation between adrenal 11ß-HSD2 expression and adrenal steroidogenic function at PW12 and PW28, and found that adrenal 11ß-HSD2 expression with adrenal NR4A1/StAR expression or serum corticosterone level were positively correlated in both male and female offspring (Fig 2e, f), suggesting that the changes in adrenal $11 \beta$-HSD2 expression affect adrenal steroidogenic function after birth in the male and female offspring.

Further, we confirm that adrenal 11ß-HSD2 expression after birth is involved in the effect of endogenous glucocorticoids (cortisol in human and corticosterone in rodents) on adrenal steroidogenic function. Firstly, we treated human adrenocortical cell line (NCI-H295R) with different concentrations (20, 100 and $500 \mathrm{nM}$ ) of cortisol, and the results showed that cortisol inhibited the mRNA expression of NR4A1 and StAR in the concentration-dependent manner (Fig 2h). Furthermore, we treated the NCI-H295R cells with 500 $\mathrm{nM}$ dexamethasone for $24 \mathrm{~h}$, and then transfected 11ß-HSD2 plasmid into the cells to increase its expression after the withdrawal of dexamethasone, which mimic in vivoexperiment. We found that dexamethasone could inhibit the expression of $11 \beta$-HSD2 in the NCI-H295R cells, and over-expression of 11 $\beta$-HSD2 significantly reversed the inhibitory effects of cortisol on the expression of NR4A1 and StAR (Fig 2i). These results 
suggested that adrenal 11 $\beta$-HSD2 is involved in the regulatory action of cortisol on intracellular steroidogenic function.

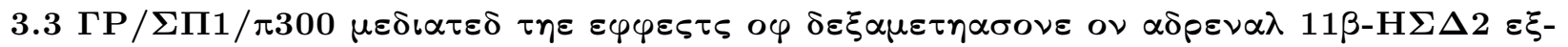

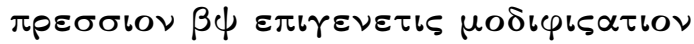

Study has confirmed that special protein 1 (SP1) can regulate the expression of 11ß-HSD2 by recruiting histone acetylase p300 (Li et al. , 2013a). We examined the expression of adrenal GR, SP1 and p300, and $\mathrm{H} 3$ acetylation of the 11 $\beta$-HSD2 promoter in the PDE offspring rats before and after birth. Compared with the control, the mRNA expression of GR increased in the male and female PDE fetal rats, the H3K14ac level of 11ß-HSD2 promoter region as well as the SP1 and p300 mRNA expression in the male PDE fetal adrenals were reduced while in the females significantly increased (Fig 2a, b), but the H3K9ac and H3K27ac levels of 11ß-HSD2 showed no significant changes at the same time (Fig 2b). At PW28, H3K9ac, H3K14ac and H3K27ac levels of adrenal 11 $\beta$-HSD2 in the male PDE offspring were significantly reduced while in the females increased (Fig 2c). These results indicated that the SP1/p300 expression and the H3K14ac level of 11ß-HSD2 in the PDE offspring showed the persistent gender difference from intrauterine to postnatal adulthood.

Furthermore, we confirmed the effects of dexamethasone on adrenal 11ß-HSD2 epigenetic modification and its expression. It is known dexamethasone can enter fetal circulation through the placenta. According to our previous data, serum dexamethasone levels of maternal, male and female fetal rats were $0.394 \mu \mathrm{g} / \mathrm{mL}$ (846 nM), $0.11 \mu \mathrm{g} / \mathrm{mL}(236 \mathrm{nM})$ (Liu et al. , 2018) and $0.124 \mu \mathrm{g} / \mathrm{mL}(267 \mathrm{nM})$, respectively (Lv et al. , 2018). Based on these, dexamethasone at different concentrations $(20,100,500 \mathrm{nM})$ was applied to the NCIH295R cells for $24 \mathrm{~h}$, and the MTS results showed no cytotoxicity (Fig 4d). We found that dexamethasone significantly increased the mRNA expression of GR (Fig 4e). Meanwhile, dexamethasone inhibited the mRNA and protein expression levels of SP1, p300, 11ß-HSD2, NR4A1, StAR, and cortisol levels in the cell supernatant in the concentration-dependent manner (Fig 4f-i), and reduced the H3K14ac level of 11 $\beta$ HSD2 (Fig 4j). Bioinformatics analysis showed that there are GR binding sites in the SP1 promoter region (-1395 to $-1381,-173$ to -159 , Fig $4 \mathrm{k})$. Therefore, we designed the primers for these predicted binding sites, and chromatin immunoprecipitation-polymerase chain reaction (ChIP-PCR) results indicated that dexamethasone $(500 \mathrm{nM})$ increased the binding of GR to the SP1 promoter region (Fig $4 \mathrm{k})$. To confirm that the GR/SP1/p300 pathway mediated the epigenetic modification and expression changes of 11ß-HSD2 induced by dexamethasone, we treated the NCI-H295R cells with GR siRNA, SP1 plasmid and CTPB (a selective activator of histone acetyltransferase p300). We found the knockdown of GR could markedly reverse the suppressive effects of dexamethasone $(500 \mathrm{nM}$ ) on SP1 expression and 11ß-HSD2 H3K14ac level (Fig 41). And the treatment with the SP1 plasmid and CTPB reversed the suppressive effects of dexamethasone (500 nM) on the H3K14ac level of 11ß-HSD2 and its expression (Fig 4m, n), and SP1 overexpression could also reversed the suppressive effect of dexamethasone on the p300 expression (Fig 4m). In conclusion, dexamethasone could inhibit SP1 expression and reduced the recruitment of p300, reducing, which cooperatively decreased the H3K14ac level and expression of 11ß-HSD2.

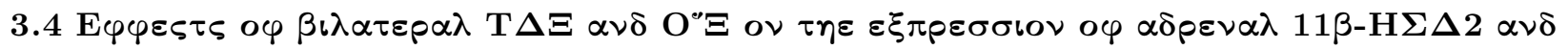
steroidogenic function in the PDE adult offspring

To investigate whether sex hormones and their receptors are involved in gender differences of adrenal 11 $\beta$ HSD2 expression and steroidogenic function by PDE, the offspring rats underwent bilateral TDX or OVX at PW22 to remove endogenous sex hormones, and the changes of adrenal 11ß-HSD2 expression and steroidogenic function were observed at PW28. Compared with the control, the mRNA expression of adrenal NR4A1 and StAR in TDX male or OVX female PDE offspring were significantly reduced $(P<0.05, P<0.01$, Fig 3a, c). Immunohistochemistry and ELISA analysis also confirmed that the adrenal NR4A1, StAR protein expression and serum corticosterone levels were also markedly reduced (Fig 3b, d, e). Meanwhile, adrenal $11 \beta$-HSD2 expression were significantly inhibited in both TDX male and OVX female PDE offspring (Fig $3 f, g)$. These results showed that TDX in males or OVX in females could eliminate the gender differences in 
adrenal 11 $\beta$-HSD2 expression and steroidogenic function in the adult PDE offspring. Moreover, we examined the expression of adrenal sex hormone receptor in the absence or presence of TDX or OVX. The mRNA expression of adrenal AR in the male adult PDE offspring was significantly decreased while adrenal ER $\beta$ in the female adult PDE offspring was significantly increased. However, after TDX or OVX, the mRNA expression of adrenal AR in the males or adrenal ER $\beta$ in the females was significantly decreased (Fig 3h), which was positively correlated with the mRNA expression of 11/-HSD2 (Fig 3i). These indicated that sex hormones and their receptors participate in the gender differences of adrenal 11ß-HSD2 expression and steroidogenic function in the PDE offspring.

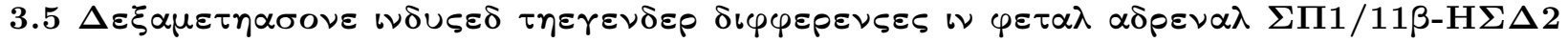

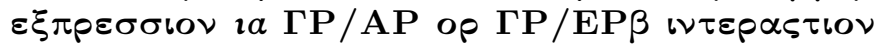

To explore the intrauterine origin mechanism of the gender differences in adrenal SP1/11/-HSD2 expression of PDE offspring, we examined the expression of adrenal sex hormone receptor and serum sex hormone level in fetal rats. We found that compared with the control, PDE reduced serum testosterone level in the males and estradiol level in the females (Fig 5a, b). However, the AR expression in the PDE males was significantly reduced, whereas the ER $\beta$ expression in the PDE females was increased (Fig 5c, d). Studies reported that AR or ER $\beta$ could induce androgen or estrogen response by binding with SP1 and forming AR/SP1 or ER $\beta / \mathrm{SP} 1$ complex (Schultz et al. , 2005; Chen et al. , 2008b). These suggested that sex hormone receptor rather than sex hormone might induce the gender differences of fetal adrenal SP1/11ß-HSD2 expression.

In order to further explore the molecular mechanism of the gender differences in SP1/11/-HSD2 expression in the PDE offspring, we treated primary fetal rat adrenal cells with $500 \mathrm{nM}$ dexamethasone, and found that the mRNA expression of GR was significantly increased in male and female fetal adrenal cells (Fig $5 \mathrm{e})$, but the mRNA expression of AR in the males was decreased and mRNA expression of ER $\beta$ in the females was increased (Fig 5f, g). We further verified the interaction between GR and AR or ER $\beta$ through Co-immunoprecipitation (Co-IP) experiments (Fig 5h). The results showed that dexamethasone activated GR in fetal adrenal cells, downregulated AR in the males and reduced the binding of GR to AR, whereas upregulated ER $\beta$ in the females and promoted the binding of GR to ER $\beta$. We further found that the SP1 and p300 expression, the H3K14ac level of 11ß-HSD2 and its expression were significantly reduced in the male fetal adrenal cells, while increased in the females (Fig 5i-l). Further, we confirmed that the AR and ER $\beta$ mediated the gender difference in SP1 expression induced by dexamethasone in the primary adrenal cells of fetal rats. We found that $10 \mathrm{nM}$ dihydrotestosterone (DHT) (AR agonist) can reverse the suppressive effect of dexamethasone on the SP1 expression in the males (Fig $5 \mathrm{~m}$ ), while ER $\beta$ siRNA can reverse the promotive effect of dexamethasone on the SP1 expression in the females (Fig 5n). These results indicated that dexamethasone activated GR in the primary fetal adrenal cells, leading to gender-specific changes in the SP1/11 $\beta$-HSD2 expression through downregulating AR in the males or upregulating ER $\beta$ in the females.

\section{Discussions}

4.1 Postnatal gender differences in adrenal steroidogenic function and its toxicologic significance in the PDE offspring rats

It is known that endocrine organs of developing individuals are highly sensitive to external environments. Glucocorticoids secreted by adrenal gland are crucial for intrauterine development and postnatal fate, while abnormal glucocorticoid levels in utero can cause catch-up growth after birth in IUGR offspring (Marciniaket al. , 2011; He et al. , 2019a). Therefore, exposure to synthetic glucocorticoids (such as dexamethasone) in early life can cause long-term toxicity by affecting the endocrine system.

Metabolic syndrome is a common disease in adults. Glucocorticoids is important hormone regulating sugar, fat, and protein metabolism in the body, and play an important role in occurrence and development of metabolic syndrome (Goodwin et al. , 2012). Therefore, the adrenal development dysfunction plays an important role in increasing offspring's susceptibility to adult metabolic syndrome, and the gender difference in adrenal steroidogenic function in offspring can cause gender differences in susceptibility to metabolic syndrome and related diseases (Facchi et al. , 2019). In our previous studies, we found that PDE induced a 
gender-difference and sustainable multi-organ damage in offspring rats via serum metabolic profile analysis (Chen et al. , 2019). Besides, other studies also demonstrated that PDE female offspring are more susceptible to metabolic disease in adulthood than males (Carbone et al. , 2012; Carpenter et al. , 2017).

Steroidogenic function is an important indicator for adrenal development. In this study, PDE inhibited adrenal steroidogenic function in male and female fetal rats, which is mainly related to the direct action of dexamethasone. Further, PDE offspring were out of dexamethasone exposure after birth, and we found that the adrenal steroidogenic function was decreased in males and increased in females, showing an obvious gender difference. This postnatal gender difference in adrenal steroidogenic function of PDE offspring may be an important reason for the gender-differentiated susceptibility of adult metabolic syndrome and related diseases.

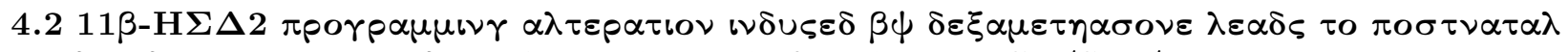
$\gamma \varepsilon \nu \delta \varepsilon \rho \delta \varphi \varphi \varphi \varepsilon \rho \varepsilon \nu \varsigma \varepsilon \iota \nu \alpha \delta \rho \varepsilon \nu \alpha \lambda$ steroidogenic function via GR/SP1/p300 pathway

$11 \beta$-HSD2 has a strong inactivation capacity against endogenous glucocorticoids (Bestet al. , 1997). Our previous study reported that adrenal 11 $\beta$-HSD2 might mediate the changes in adrenal steroidogenic function induced by maternal glucocorticoid through inactivating locally active glucocorticoids in offspring rats with prenatal caffeine or ethanol exposure (Huang et al. , 2015; He et al. , 2019b). In this study, we found that there was a consistent gender difference in adrenal 11ß-HSD2 expression and steroidogenic function of PDE offspring after birth, which decreased in the males while increased in the females. Interestingly, we demonstrated that the expression change of adrenal 11ß-HSD2 was from the intrauterine period, and there were significant positive correlations between adrenal 11 $\beta$-HSD2 expression and steroidogenic functionrelated indicators after birth. All the results suggested that 11ß-HSD2 is involved in regulating the gender difference in postnatal adrenal steroidogenic function caused by PDE.

We confirmed that cortisol inhibited the expression of NR4A1 and StAR in the concentration-dependent manner in NCI-H295R cells. Further, after pretreated with dexamethasone, 11ß-HSD2 overexpression could significantly reverse the inhibition of cortisol on the NR4A1/StAR signal, which indicated that 11 $\beta$-HSD2 affects postnatal adrenal steroidogenic function by metabolizing endogenous glucocorticoids in the adult PDE offspring. Therefore, postnatal abnormal adrenal steroidogenic function in the PDE offspring was related to the local CORT level regulated by the metabolism of 113-HSD2, and the intrauterine programming alteration of adrenal 11ß-HSD2 determined the gender difference of adrenal steroidogenic function in the PDE offspring after birth.

Epigenetic modification participates in multi-organ life programming and homeostasis, which plays a vital role in occurrence and development of fetal-originated adult diseases (Moisiadis et al. , 2014b). Epigenetic regulations in the early development period can persistently mediate phenotypic changes in later life. Among them, histone modification is mainly involved in the regulation of gene transcriptional expression (Huan et al. , 2015). A study in the Netherlands showed that epigenetic modification changes in offspring caused by famine can occur in a sex-specific manner (Tobi et al. , 2009). In this study, we screened different histone acetylation sites in the promoter region of adrenal 11ß-HSD2 before and after birth in the PDE offspring, and found only H3K14ac levels were consistent with the expression of 11ß-HSD2. That is, the H3K14ac levels of adrenal 11ß-HSD2 and its expression decreased in the males, but increased in the females. Moreover, in the NCI-H295R cells, we demonstrated that dexamethasone treatment reduced the H3K14ac level of 11 $\beta$ HSD2 and its expression. These suggested that the H3K14ac level changes of adrenal 11ß-HSD2 induced by dexamethasone mediated the consistent changes of its expression before and after birth.

As an essential transcriptional activator of $11 \beta-H S D 2$, SP1 can alter histone acetylation of the 11ß-HSD2 promoter region by recruiting coactivator-histone acetylase p300 and promote its expression (Liet al. , 2013b). In the NCI-H295R cells, we confirmed that dexamethasone increased the expression and nuclear translocation of GR, inhibited the expression of SP1 and p300, and reduced the H3K14ac level and expression of $11 \beta$-HSD2. We also demonstrated that dexamethasone increased the binding of GR to the SP1 promoter region. Furthermore, the GR siRNA, SP1 plasmid or p300 activator was proved to reverse the expression 
inhibition of dexamethasone on the respective downstream gene SP1, p300 and 11ß-HSD2. The above results suggested that the GR/SP1/p300 pathway mediated the epigenetic regulation of dexamethasone on the $11 \beta$-HSD2 expression in adrenocortical cells.

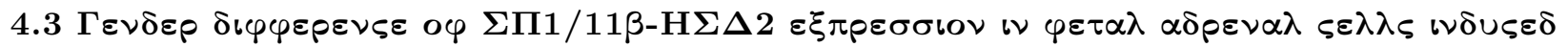

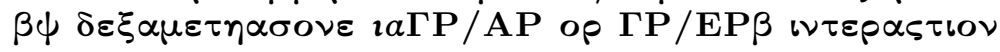

Studies suggested that sex hormones and their receptors might be involved in regulating adrenal development (Hirst et al. , 1992; Albrecht et al. , 1999; Bhasin et al. , 2007). We performed bilateral TDX or OVX in male and female offspring of PW22 rats to remove the effects of endogenous sex hormones. We found the expression of adrenal both AR in males or ER $\beta$ in females of the PDE offspring were significantly reduced; meanwhile, the adrenal 11ß-HSD2 expression and steroidogenic function were also markedly decreased. Moreover, AR expression in the males or ER $\beta$ expression in the females was positively correlated with 11ß-HSD2 expression. These results suggested that the gender differences in adrenal 11/-HSD2 expression and steroidogenic function might be related to sex hormones and their receptors in the adult PDE offspring.

Then, we explored the sex hormones and/or their receptors-related mechanism of the gender difference in adrenal 11 $\beta$-HSD2 expression and its intrauterine origin in the PDE offspring. We found that serum testosterone level in the male PDE fetal rats and estradiol level in the females was reduced, but adrenal AR expression was decreased in the males while ER $\beta$ expression was increased in the females. Studied suggested that the effect of glucocorticoids on androgen or estrogen action is mediated through regulating AR or ER level (Smith et al. , 1984; Kamble et al. , 2019). Dexamethasone can down-regulate AR mRNA and protein, and may thereby influence androgen responsiveness (Burnstein et al. , 1995). Dexamethasone can also affect estrogen reactivity by increasing ER $\beta$ mRNA level by GR binding to the glucocorticoid binding element (GRE) of the ER promoter region (Miranda et al. , 2013). We further confirmed that dexamethasone reduced the binding of GR with AR (GR/AR interaction) in the males while increased the binding of GR with ER $\beta$ (GR/ER $\beta$ interaction) in the females. The above results suggested sex hormone receptors rather than sex hormones induced the gender difference in fetal adrenal 11 $\beta$-HSD2 expression.

It is known that SP1 is a critical transcriptional activator of 11/-HSD2, both AR and ER $\beta$ can interact with SP1 and bind to the downstream target gene 11ß-HSD2 through SP1, thus regulating its expression (Saville et al. , 2000; Chen et al. , 2008a). To confirm that gender differences in adrenal SP1/11ß-HSD2 expression are associated with sex hormone receptors, we isolated the adrenal cells of normal fetal rats. We confirmed dexamethasone activated fetal adrenal GR, down-regulating the expression and binding of AR to GR in the male primary adrenal cells, or up-regulating the expression and binding of ER $\beta$ to GR in the females. It differentially regulated the expression of SP1, eventually resulting in gender differences in the H3K14ac level of $11 \beta$-HSD2 and its expression (Fig 6).

\subsection{The basis for the establishment of PDE rat model in the present study}

In the study, the time and dose selection of dexamethasone were based on the following: according to the recommendation of the WHO, the classic clinical use of dexamethasone for preventive treatment of premature is an intramuscular injection every 12 hours, $6 \mathrm{mg}$ each time for a total of 4 times, with the optimal time in gestational 24 to 34 weeks. However, due to the difficulty in early diagnosis of preterm labor and the poor response to single administration in some pregnant women, approximately $1 / 3$ of pregnant women changed from preventive to continuous administration (Murphy et al. , 2008), even more than 11 courses (Quinlivan et al. , 1998), so multi-course treatment of dexamethasone is possible in the clinical practice. Besides, the development of rat adrenal cortex begins with the migration of epithelial cells from the coelomic epithelium of the urogenital system on GD9 (Hatano et al. , 1996). Therefore, we established a rat model of PDE in the middle and late trimester. It's known that the dose conversion coefficient between rats and humans was $6.16: 1$ in pharmacological experiments, so $0.2 \mathrm{mg} / \mathrm{kg} \cdot \mathrm{d}$ dexamethasone in rats was equal to $0.03 \mathrm{mg} / \mathrm{kg} \cdot \mathrm{d}$ in humans, while the clinical standard dose of dexamethasone for preterm labor is $0.05-0.2 \mathrm{mg} / \mathrm{kg} \cdot \mathrm{d}$ (Moisiadis et al. , 2014a). Therefore, the dose of dexamethasone in this study is lower than the clinical dose.

We would like to admit that in this study, the time and dose selection of dexamethasone exposure during 
pregnancy cannot fully mimic the clinical administration, this is the limitation of PDE rat model application of this study. It is worth mentioning that, in previous studies, we have demonstrated that PDE could dosedependently reduce birth weight and increase the IUGR rate, and its toxic effects on fetal development was more obvious in the second trimester than in the third trimester (Chen et al. , 2018b). Therefore, in this study, using this stably established PDE rat model, we confirmed the changes in adrenal steroidogenic function of PDE offspring and their gender differences, and elucidated its intrauterine epigenetic programming mechanism, which is of great practical significance for exploring the early intervention measures of fetaloriginated diseases.

\section{Conclusions}

This study firstly confirmed PDE-induced adrenal developmental toxicity, long-term harm and its gender difference, and elucidated the 11 $\beta$-HSD2-mediated intrauterine epigenetic programming mechanism. That is, dexamethasone by activating fetal adrenal GR, down-regulated AR expression and reduced the binding of AR with GR in the male offspring, while up-regulated ER $\beta$ expression and increased the binding of ER $\beta$ with GR in the females, which caused the gender difference in the H3K14ac level of 11ß-HSD2 and its expression via GR/SP1/p300 pathway, and eventually leading to the gender difference in adrenal steroidogenic function of PDE offspring after birth (Fig 7). This probably is one of the main reasons for the gender differences in fetal-originated metabolic syndrome and related diseases induced by PDE. This study is also the first time to explaining the gender difference in the fetal-originated diseases from the perspective of the intrauterine origin of adrenal development, and will provide experimental evidence and new academic viewpoint for exploring early prevention and treatment measures.

\section{Declaration of transparency and scientific rigour}

This Declaration acknowledges that this paper adheres to the principles for transparent reporting and scientific rigour of preclinical research as stated in the BJP guidelines for Design \& Analysis, Immunoblotting and Immunochemistry and Animal Experimentation, and as recommended by funding agencies, publishers, and other organizations engaged with supporting research.

\section{References:}

Albrecht ED, Babischkin JS, Davies WA, Leavitt MG, Pepe GJ (1999). Identification and developmental expression of the estrogen receptor alpha and beta in the baboon fetal adrenal gland. Endocrinology 140 (12): 5953-5961.

Arnetz L, Rajamand Ekberg N, Brismar K, Alvarsson M (2015). Gender difference in adrenal sensitivity to ACTH is abolished in type 2 diabetes. Endocr connect4 (2): 92-99.

Ayonrinde OT, Olynyk JK, Beilin LJ, Mori TA, Pennell CE, de Klerk N, et al. (2011). Gender-specific differences in adipose distribution and adipocytokines influence adolescent nonalcoholic fatty liver disease. Hepatology 53 (3): 800-809.

Bailey MA (2017). 11beta-hydroxysteroid dehydrogenases and hypertension in the metabolic syndrome. Curr. Hypertens. Rep. 19 (12): 100.

Best R, Nelson SM, Walker BR (1997). Dexamethasone and 11-dehydrodexamethasone as tools to investigate the isozymes of 11 beta-hydroxysteroid dehydrogenase in vitro and in vivo.J. Endocrinol. 153 (1): 41-48.

Bhasin S, Enzlin P, Coviello A, Basson R (2007). Sexual dysfunction in men and women with endocrine disorders.Lancet 369 (9561): 597-611.

Burnstein KL, Maiorino CA, Dai JL, Cameron DJ (1995). Androgen and glucocorticoid regulation of androgen receptor cDNA expression. Mol. Cell. Endocrinol.115 (2): 177-186.

Carbone DL, Zuloaga DG, Hiroi R, Foradori CD, Legare ME, Handa RJ (2012). Prenatal dexamethasone exposure potentiates diet-induced hepatosteatosis and decreases plasma IGF-I in a sex-specific fashion. Endocrinology 153 (1):295-306. 
Carpenter T, Grecian SM, Reynolds RM (2017). Sex differences in early-life programming of the hypothalamic-pituitary-adrenal axis in humans suggest increased vulnerability in females: a systematic review. J. Dev. Orig. Health Dis. 8 (2): 244-255.

Chapman K, Holmes M, Seckl J (2013). 11beta-hydroxysteroid dehydrogenases: intracellular gate-keepers of tissue glucocorticoid action. Physiol. Rev.93 (3): 1139-1206.

Chen G, Xiao H, Zhang J, Zhang H, Li B, Jiang T, et al. (2019). Prenatal dexamethasone exposure-induced a gender-difference and sustainable multi-organ damage in offspring rats via serum metabolic profile analysis. Toxicol. Lett. 316: 136-146.

Chen G, Yuan C, Duan F, Liu Y, Zhang J, He Z, et al. (2018a). IGF1/MAPK/ERK signaling pathwaymediated programming alterations of adrenal cortex cell proliferation by prenatal caffeine exposure in male offspring rats. Toxicol. Appl. Pharmacol. 341: 64-76.

Chen P-H, Tsao Y-P, Wang C-C, Chen S-L (2008a). Nuclear receptor interaction protein, a coactivator of androgen receptors (AR), is regulated by $\mathrm{AR}$ and $\mathrm{Sp} 1$ to feed forward and activate its own gene expression through AR protein stability.Nucleic Acids Res. 36 (1): 51-66.

Chen PH, Tsao YP, Wang CC, Chen SL (2008b). Nuclear receptor interaction protein, a coactivator of androgen receptors (AR), is regulated by $\mathrm{AR}$ and $\mathrm{Sp} 1$ to feed forward and activate its own gene expression through AR protein stability. Nucleic Acids Res. 36 (1): 51-66.

Chen Z, Zhao X, Li Y, Zhang R, Nie Z, Cheng X, et al. (2018b). Course-, dose-, and stage-dependent toxic effects of prenatal dexamethasone exposure on long bone development in fetal mice. Toxicol. Appl. Pharmacol.351: 12-20.

Dasinger JH, Alexander BT (2016). Gender differences in developmental programming of cardiovascular diseases. Clinical science 130 (5): 337-348.

Doyle LW, Ford GW, Davis NM, Callanan C (2000). Antenatal corticosteroid therapy and blood pressure at 14 years of age in preterm children. Clin Sci (Lond) 98 (2): 137-142.

Facchi JC, de Lima TAL, de Oliveira LR, de Oliveira Costermani H, de Souza Miranda GD, de Oliveira JC (2019). Perinatal programming of metabolic diseases: the role of glucocorticoids. Metabolism : 154047.

Goodwin JE, Geller DS (2012). Glucocorticoid-induced hypertension. Pediatr. Nephrol.27 (7): 1059-1066.

Hatano O, Takakusu A, Nomura M, Morohashi K (1996). Identical origin of adrenal cortex and gonad revealed by expression profiles of Ad4BP/SF-1. Genes Cells 1 (7): 663-671.

He B, Wen Y, Hu S, Wang G, Hu W, Magdalou J, et al. (2019a). Prenatal caffeine exposure induces liver developmental dysfunction in offspring rats. J. Endocrinol.242 (3): 211-226.

He Z, Zhang J, Huang H, Yuan C, Zhu C, Magdalou J, et al. (2019b). Glucocorticoid-activation system mediated glucocorticoid-insulin-like growth factor 1 (GC-IGF1) axis programming alteration of adrenal dysfunction induced by prenatal caffeine exposure. Toxicol. Lett. 302: 7-17.

Hirst JJ, West NB, Brenner RM, Novy MJ (1992). Steroid hormone receptors in the adrenal glands of fetal and adult rhesus monkeys. J. Clin. Endocrinol. Metab.75 (1): 308-314.

Huan Y, Wu Z, Zhang J, Zhu J, Liu Z, Song X (2015). Epigenetic Modification Agents Improve Gene-Specific Methylation Reprogramming in Porcine Cloned Embryos. PloS one10 (6): e0129803.

Huang H, He Z, Zhu C, Liu L, Kou H, Shen L, et al. (2015). Prenatal ethanol exposure-induced adrenal developmental abnormality of male offspring rats and its possible intrauterine programming mechanisms. Toxicol. Appl. Pharmacol.288 (1): 84-94. 
Kamble PG, Pereira MJ, Almby K, Eriksson JW (2019). Estrogen interacts with glucocorticoids in the regulation of lipocalin 2 expression in human adipose tissue. Reciprocal roles of estrogen receptor $\alpha$ and $\beta$ in insulin resistance? Mol. Cell. Endocrinol. 490: 28-36.

Li J, Wang W, Liu C, Wang W, Li W, Shu Q, et al. (2013a). Critical Role of Histone Acetylation by p300 in Human Placental 11ß-HSD2 Expression. The Journal of Clinical Endocrinology 83 Metabolism 98 (7):E1189-E1197.

Li J, Wang W, Liu C, Wang W, Li W, Shu Q, et al. (2013b). Critical role of histone acetylation by p300 in human placental 11beta-HSD2 expression. J. Clin. Endocrinol. Metab. 98 (7): E1189-1197.

Liu M, Chen B, Pei L, Zhang Q, Zou Y, Xiao H, et al. (2018). Decreased H3K9ac level of StAR mediated testicular dysplasia induced by prenatal dexamethasone exposure in male offspring rats. Toxicology 408: $1-10$.

Long NM, Shasa DR, Ford SP, Nathanielsz PW (2012). Growth and insulin dynamics in two generations of female offspring of mothers receiving a single course of synthetic glucocorticoids. American journal of obstetrics and gynecology 207 (3): 203 e201-208.

Lv F, Wan Y, Chen Y, Pei L, Luo D, Fan G, et al. (2018). Prenatal Dexamethasone Exposure Induced Ovarian Developmental Toxicity and Transgenerational Effect in Rat Offspring. Endocrinology 159 (3): 1401-1415.

Marciniak B, Patro-Malysza J, Poniedzialek-Czajkowska E, Kimber-Trojnar Z, Leszczynska-Gorzelak B, Oleszczuk J (2011). Glucocorticoids in pregnancy. Current pharmaceutical biotechnology 12 (5): 750-757.

Masuzaki H, Paterson J, Shinyama H, Morton NM, Mullins JJ, Seckl JR, et al. (2001). A transgenic model of visceral obesity and the metabolic syndrome. Science 294 (5549): 2166-2170.

McNeil CJ, Nwagwu MO, Finch AM, Page KR, Thain A, McArdle HJ, et al. (2007). Glucocorticoid exposure and tissue gene expression of 11beta HSD-1, 11beta HSD-2, and glucocorticoid receptor in a porcine model of differential fetal growth.Reproduction (Cambridge, England) 133 (3):653-661.

Millership SJ, Van de Pette M, Withers DJ (2019). Genomic imprinting and its effects on postnatal growth and adult metabolism. Cell. Mol. Life Sci.76 (20): 4009-4021.

Miranda TB, Voss TC, Sung MH, Baek S, John S, Hawkins M, et al. (2013). Reprogramming the chromatin landscape: interplay of the estrogen and glucocorticoid receptors at the genomic level. Cancer Res. $\mathbf{7 3}$ (16): 5130-5139.

Moisiadis VG, Matthews SG (2014a). Glucocorticoids and fetal programming part 1: Outcomes. Nat. Rev. Endocrinol. 10 (7): 391-402.

Moisiadis VG, Matthews SG (2014b). Glucocorticoids and fetal programming part 2: Mechanisms. Nat. Rev. Endocrinol. 10 (7): 403-411.

Murphy KE, Hannah ME, Willan AR, Hewson SA, Ohlsson A, Kelly EN, et al. (2008). Multiple courses of antenatal corticosteroids for preterm birth (MACS): a randomised controlled trial. Lancet 372 (9656): 2143-2151.

O'Connell BA, Moritz KM, Walker DW, Dickinson H (2013). Treatment of pregnant spiny mice at mid gestation with a synthetic glucocorticoid has sex-dependent effects on placental glycogen stores. Placenta 34 (10): 932-940.

Ojeda NB, Intapad S, Alexander BT (2014). Sex differences in the developmental programming of hypertension. Acta physiologica 210 (2):307-316.

Pawluski JL, Brain UM, Underhill CM, Hammond GL, Oberlander TF (2012). Prenatal SSRI exposure alters 
neonatal corticosteroid binding globulin, infant cortisol levels, and emerging HPA function. Psychoneuroendocrinology 37 (7):1019-1028.

Pinheiro CR, Oliveira E, Trevenzoli IH, Manhaes AC, Santos-Silva AP, Younes-Rapozo V, et al. (2011). Developmental plasticity in adrenal function and leptin production primed by nicotine exposure during lactation: gender differences in rats. Horm. Metab. Res. 43 (10): 693-701.

Quinlivan JA, Evans SF, Dunlop SA, Beazley LD, Newnham JP (1998). Use of corticosteroids by Australian obstetricians-a survey of clinical practice. Aust. N. Z. J. Obstet. Gynaecol. 38 (1): 1-7.

Roberts D, Brown J, Medley N, Dalziel SR (2017). Antenatal corticosteroids for accelerating fetal lung maturation for women at risk of preterm birth. The Cochrane database of systematic reviews 3: Cd004454.

Saville B, Wormke M, Wang F, Nguyen T, Enmark E, Kuiper G, et al. (2000). Ligand-, cell-, and estrogen receptor subtype (alpha/beta)-dependent activation at GC-rich (Sp1) promoter elements. J. Biol. Chem. 275 (8):5379-5387.

Schaafsma SM, Gagnidze K, Reyes A, Norstedt N, Mansson K, Francis K, et al. (2017). Sex-specific gene-environment interactions underlying ASD-like behaviors. Proc. Natl. Acad. Sci. U. S. A. 114 (6): 1383-1388.

Schultz JR, Petz LN, Nardulli AM (2005). Cell- and ligand-specific regulation of promoters containing activator protein-1 and Sp1 sites by estrogen receptors alpha and beta.J. Biol. Chem. 280 (1): 347-354.

Smith RG, Syms AJ, Norris JS (1984). Differential effects of androgens and glucocorticoids on regulation of androgen receptor concentrations and cell growth. J. Steroid Biochem. 20 (1): 277-281.

Tobi EW, Lumey LH, Talens RP, Kremer D, Putter H, Stein AD, et al. (2009). DNA methylation differences after exposure to prenatal famine are common and timing- and sex-specific. Hum. Mol. Genet. 18 (21):40464053.

Tomaszewska E, Dobrowolski P, Siwicki A (2012). Maternal treatment with dexamethasone at minimal therapeutic doses inhibits neonatal bone development in a gender-dependent manner.Livest Sci 146 (2-3): 175-182.

Trautman PD, Meyer-Bahlburg HF, Postelnek J, New MI (1995). Effects of early prenatal dexamethasone on the cognitive and behavioral development of young children: results of a pilot study. Psychoneuroendocrinology 20 (4):439-449.

van Abeelen AF, de Rooij SR, Osmond C, Painter RC, Veenendaal MV, Bossuyt PM, et al. (2011). The sex-specific effects of famine on the association between placental size and later hypertension. Placenta 32 (9):694-698.

Waddell BJ, Bollen M, Wyrwoll CS, Mori TA, Mark PJ (2010). Developmental programming of adult adrenal structure and steroidogenesis: effects of fetal glucocorticoid excess and postnatal dietary omega-3 fatty acids. J. Endocrinol.205 (2): 171-178.

Xu YJ, Sheng H, Wu TW, Bao QY, Zheng Y, Zhang YM, et al. (2018). CRH/CRHR1 mediates prenatal synthetic glucocorticoid programming of depression-like behavior across 2 generations. FASEB J. 32 (8): 4258-4269.

Yun HJ, Lee JY, Kim MH (2016). Prenatal exposure to dexamethasone disturbs sex-determining gene expression and fetal testosterone production in male embryos. Biochemical and biophysical research communications471 (1): 149-155.

Figure legends

Fig 1. Changes in body weights and adrenal steroidogenic function in PDE fetal and adult offspring rats. (a) Body weight on GD20; (b) IUGR rate; (c) Serum CORT concentration on GD 20; (d, e) 
steroidogenesis-related genes mRNA expression on GD20; (f, g) Adrenal NR4A1 and StAR protein expression on GD20 (yellow represents positive cells, 400×); (h, k) Serum CORT concentrations at PW12 and PW28; (i, $\mathrm{j}, \mathrm{l}, \mathrm{m}$ ) Adrenal steroidogenesis-related genes mRNA expression at PW12 and PW28; (n, o) Adrenal NR4A1 and StAR protein expression at PW28 (yellow represents positive cells, $400 \times$ ) $. \mathrm{n}=8$ from 8 different litters for body weight, IUGR rate, serum CORT concentration detection and RT-qPCR; $\mathrm{n}=5$ from 5 different litters for IHC in each group. The average weight of each litter was calculated and was thought as a single $\mathrm{n}$ value; adrenal or serum samples of 3 fetal rats from the same litter were combined into one sample, respectively. Comparisons between groups were performed using Student's $t$-test or Chi-square test. Mean \pm S.E.M. ${ }^{*} P<0.05,{ }^{* *} P<0.01$ vs . respective controls. PDE, prenatal dexamethasone exposure; GD, gestational day; PW, postnatal week; IUGR, intrauterine growth retardation; CORT, corticosterone; NR4A1, nuclear receptor subfamily 4 group A member 1; StAR, steroidogenic acute regulatory protein; P450scc, cytochrome P450 cholesterol side-chain cleavage; $3 \beta$-HSD, 3 $\beta$-hydroxysteroid dehydrogenase; P450c21, steroid 21-hydroxylase; P450c11, steroid 11ß-hydroxylase.

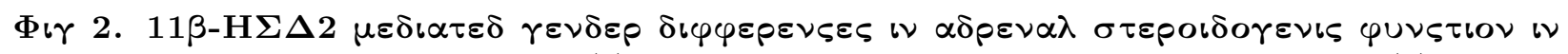

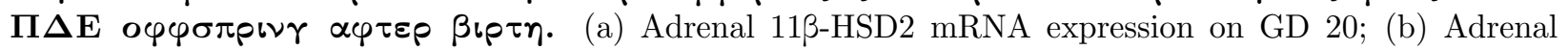

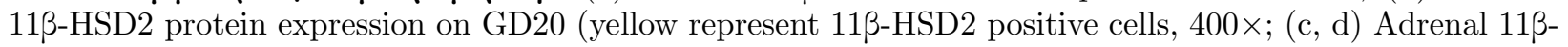
HSD2 mRNA expression at PW 12 and PW28; (e,f) The correlations between mRNA expression of adrenal

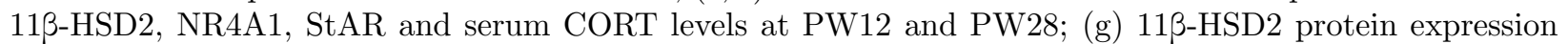
at PW28 (yellow represents 11 $\beta$-HSD2 positive cells, 400×); (h) NR4A1 and StAR mRNA expression with cortisol treatment; (i) NR4A1 and StAR mRNA expression in the presence of $11 \beta$-HSD2 plasmid. $\mathrm{n}=8$ from 8 different litters for RT-qPCR, $\mathrm{n}=5$ from 5 different litters for IHC, $\mathrm{n}=6$ for RT-qPCR from triplicate independent cell experiments in each group. Adrenal samples of 3 fetal rats from the same litter were combined into one sample. Comparisons between groups were performed using Student'st -test. Mean \pm S.E.M. ${ }^{*} P<0.05,{ }^{* *} P<0.01$ vs . respective controls. 11ß-HSD2, hydroxysteroid 11-beta dehydrogenase 2 ; PDE, prenatal dexamethasone exposure; GD, gestational day; PW, postnatal week; IUGR, intrauterine growth retardation; CORT, corticosterone; NR4A1, nuclear receptor subfamily 4 group A member 1; StAR, steroidogenic acute regulatory protein.

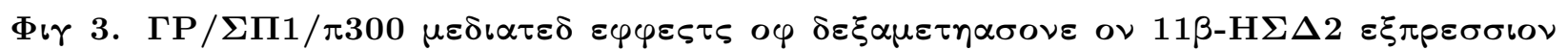

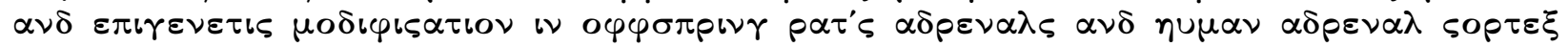
$\varsigma \varepsilon \lambda \lambda \varsigma$. (a) GR, SP1 and p300 mRNA expression in the PDE offspring; (b, c) Enrichment of H3K9ac, H3K14ac and H3K27ac at the promoter region of 11ß-HSD2 on GD20and PW28, preimmune IgG served as the negative control; (d) Cytotoxicity of dexamethasone by MTS assay; (e-g) GR, SP1, p300, 11ß-HSD2 NR4A1 and StAR mRNA expression; (h) SP1, p300, 11/-HSD2, NR4A1 and StAR protein expression; (i) Cortisol levels in cell supernatant; (j) Enrichment of H3K14ac at the promoter region of 11 $\beta$-HSD2 gene; (k) Analysis of SP1 promoter region and detection of GR binding to SP1 promoter region; (l) SP1 mRNA expression and enrichment of H3K14ac at the 11ß-HSD2 promoter region with GR siRNA; (m) p300 and $11 \beta$-HSD2 expression, and enrichment of H3K14ac at the 11ß-HSD2 promoter region with transfection of SP1 plasmid; (n) 11 $\beta$-HSD2 mRNA and protein expression and enrichment of H3K14ac at the 11 $\beta$-HSD2 promoter region with transfection in the presence of the specific activator of p300, CTPB. $\mathrm{n}=8$ from 8 different litters for RT-qPCR, $\mathrm{n}=6$ from 6 different litters for ChIP assay, $\mathrm{n}=5$ for Western blot, $\mathrm{n}=6$ for RT-qPCR from triplicate independent cell experiments in each group. Adrenal samples of 3 fetal rats from the same litter were combined into one sample. Comparisons between groups were performed using Student's $t$ -test and ANOVA. Mean \pm S.E.M., ${ }^{*} P<0.05,{ }^{* *} P<0.01$ vs. respective controls. GR, glucocorticoid receptor; SP1, special protein 1; 11ß-HSD2, hydroxysteroid 11-beta dehydrogenase 2; PDE, prenatal dexamethasone exposure; NR4A1, nuclear receptor subfamily 4 group A member 1; StAR, steroidogenic acute regulatory protein; H3K14ac, histone 3 lysine 14 acetylation; CTPB, N-(4-chloro-3-trifluoromethyl-phenyl)-2-ethoxy-6pentadecyl-benzamide.

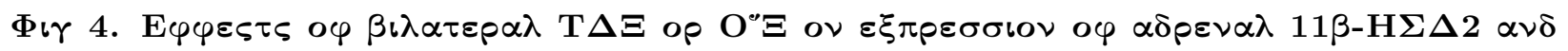

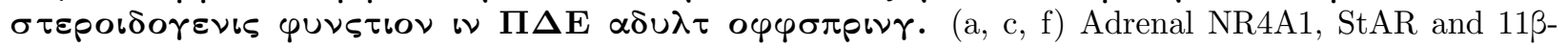
HSD2 mRNA expression in TDX or OVX adult offspring rats; (b, d, g) Adrenal NR4A1, StAR and 11 $\beta$-HSD2 
protein expression in TDX or OVX adult offspring rats at PW28 (yellow represents positive cells, 400×); (e) Serum CORT levels in TDX or OVX adult offspring rats; (h) Adrenal AR and ER $\beta$ mRNA expression in adult offspring rats without or with TDX/OVX; (i) The correlation analysis of adrenal AR or ER $\beta$ mRNA expression and 11ß-HSD2 in adult offspring rats with TDX/OVX and without TDX/OVX. $\mathrm{n}=6$ from 6 different litters for RT-qPCR and serum CORT concentration detection in adult offspring rats with TDX or OVX, $\mathrm{n}=8$ from 8 different litters for RT-qPCR in adult offspring rats without TDX or OVX, $\mathrm{n}=5$ from 5 different litters for IHC in each group. Comparisons between groups were performed using Student's $t$-test. Mean \pm S.E.M. ${ }^{*} P<0.05,{ }^{* *} P<0.01$ vs . respective controls. TDX, testectomy; OVX, ovariectomy; $11 \beta$-HSD2, hydroxysteroid 11-beta dehydrogenase 2; PDE, prenatal dexamethasone exposure; PW, postnatal week; CORT, corticosterone; NR4A1, nuclear receptor subfamily 4 group A member 1; StAR, steroidogenic acute regulatory protein; AR, androgen receptor; ER $\beta$, estrogen receptor beta.

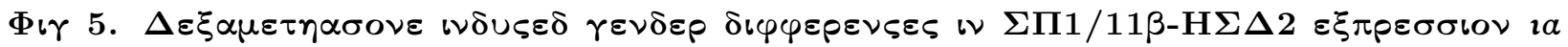
ГP/AP op ГP/EP $\alpha \delta \rho \varepsilon \nu \alpha \lambda \zeta \varepsilon \lambda \lambda \varsigma$. (a) Serum testosterone concentration in the PDE male offspring; (b) Serum estradiol concentration in the PDE female offspring; (c) Arenal AR mRNA expression in the PDE male offspring; (d) ER $\beta$ mRNA expression in the PDE female offspring; (e) GR mRNA expression in the primary fetal adrenal cells; (f) AR mRNA expression in the male primary fetal adrenal cells; (g) ER $\beta$ mRNA expression in the female primary fetal adrenal cells; (h) Co-IP assay of GR and AR or ER $\beta$ in the primary fetal adrenal cells; (i, m, n) SP1 mRNA expression in the primary fetal adrenal cells; (j) p300 mRNA expression in the primary fetal adrenal cells; (k, l) The enrichment of H3K14ac and expression of 11ß-HSD2 in the primary fetal adrenal cells. $\mathrm{n}=8$ from 8 different litters for serum testosterone, estradiol concentration detection and RT-qPCR, $\mathrm{n}=6$ from 6 different litters for ChIP assay, $\mathrm{n}=5$ for Western blot, $\mathrm{n}=6$ for RT-qPCR from triplicate independent cell experiments in each group. Adrenal or serum samples of 3 fetal rats from the same litter were combined into one sample, respectively; primary fetal adrenal cells of 10 fetal rats from the same litter were combined into one sample. Comparisons between groups were performed using Student's $t$ -test and ANOVA. Mean \pm S.E.M. ${ }^{*} P<0.05,{ }^{* *} P<0.01$ vs . respective controls. GR, glucocorticoid receptor; $\mathrm{SP} 1$, special protein 1; AR, androgen receptor; ER $\beta$, estrogen receptor beta, PDE, prenatal dexamethasone exposure.

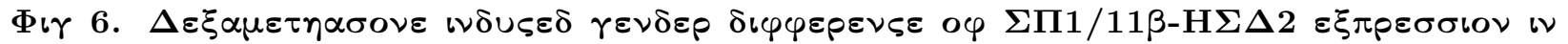
$\varphi \varepsilon \tau \alpha \lambda \alpha \delta \rho \varepsilon \nu \alpha \lambda \varsigma \varepsilon \lambda \lambda \varsigma \imath$ \а glucocorticoid receptor element; SP1, special protein 1; 11 $\beta$-HSD2, hydroxysteroid 11-beta dehydrogenase 2; H3K14ac, histone 3 lysine 14 acetylation; AR, androgen receptor; ER $\beta$, estrogen receptor beta; ARE, androgen binding element; ERE, estrogen binding element.

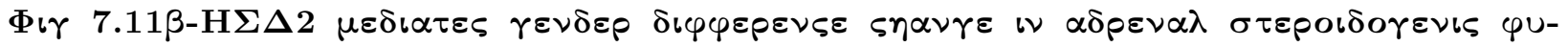

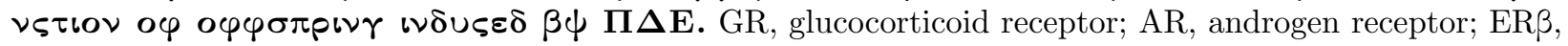
estrogen receptor beta; SP1, special protein 1; H3K14ac, histone 3 lysine 14 acetylation. 


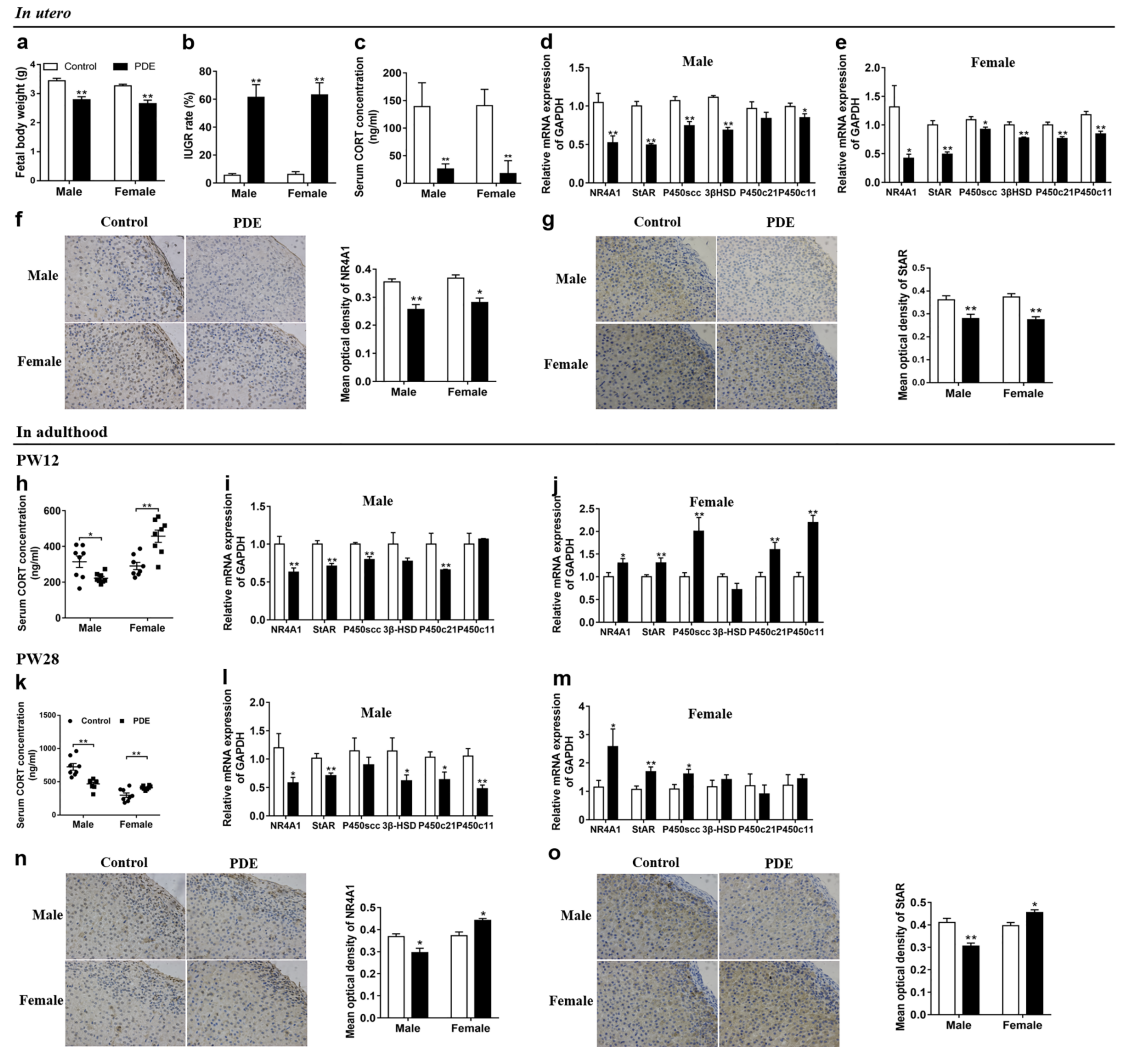




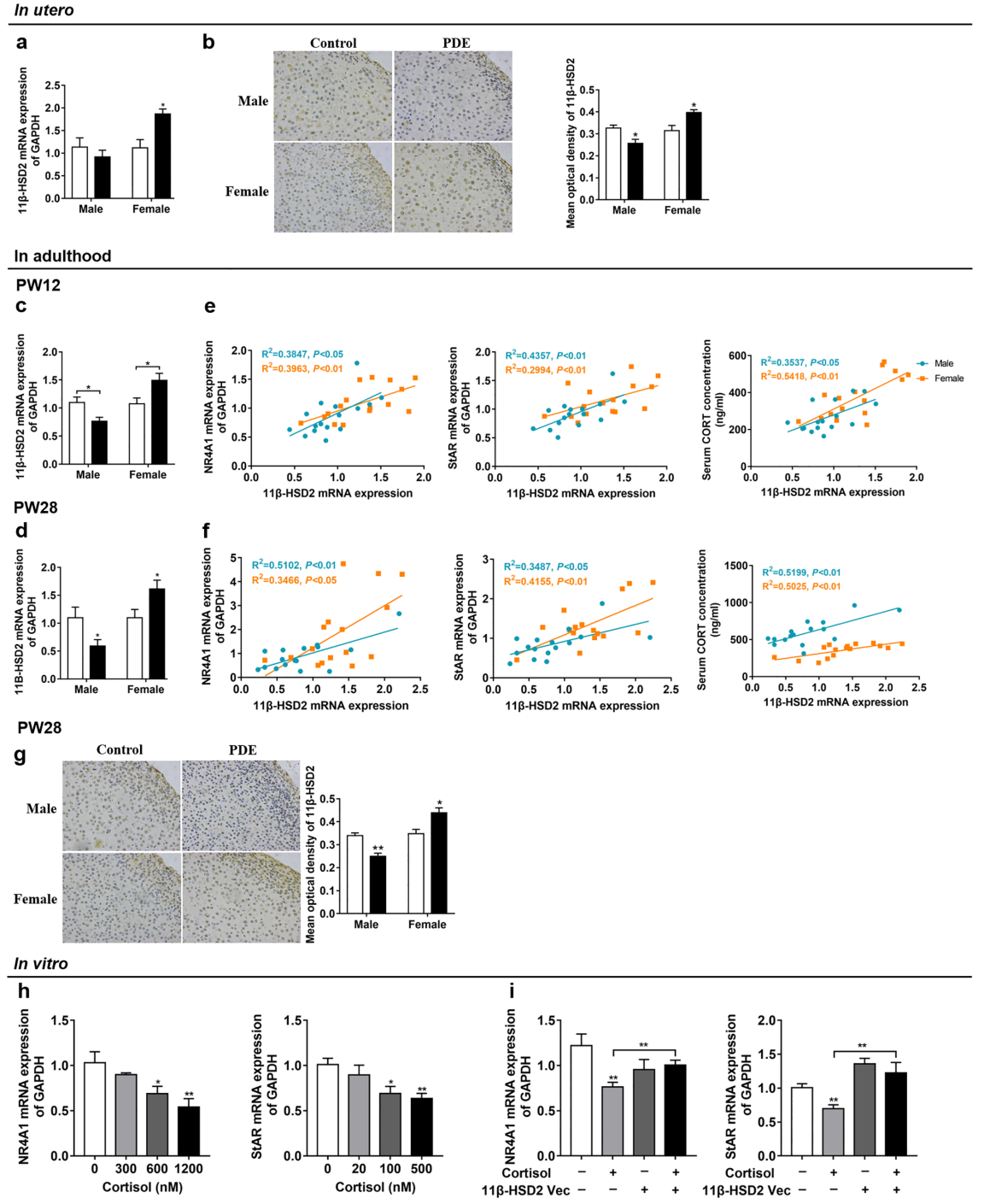




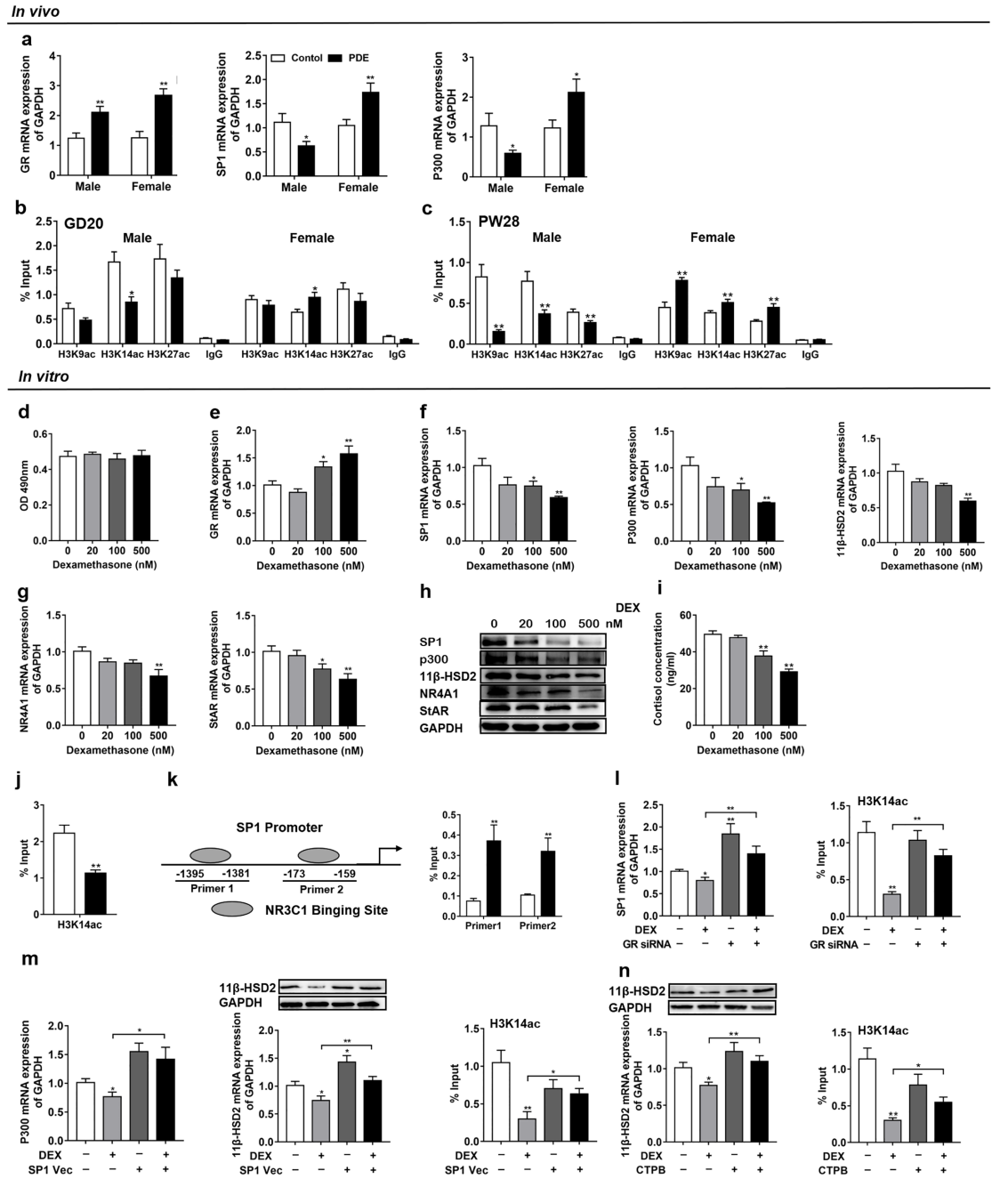


a

b
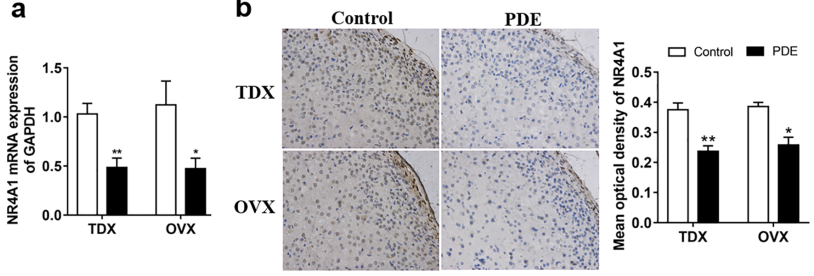

C

d
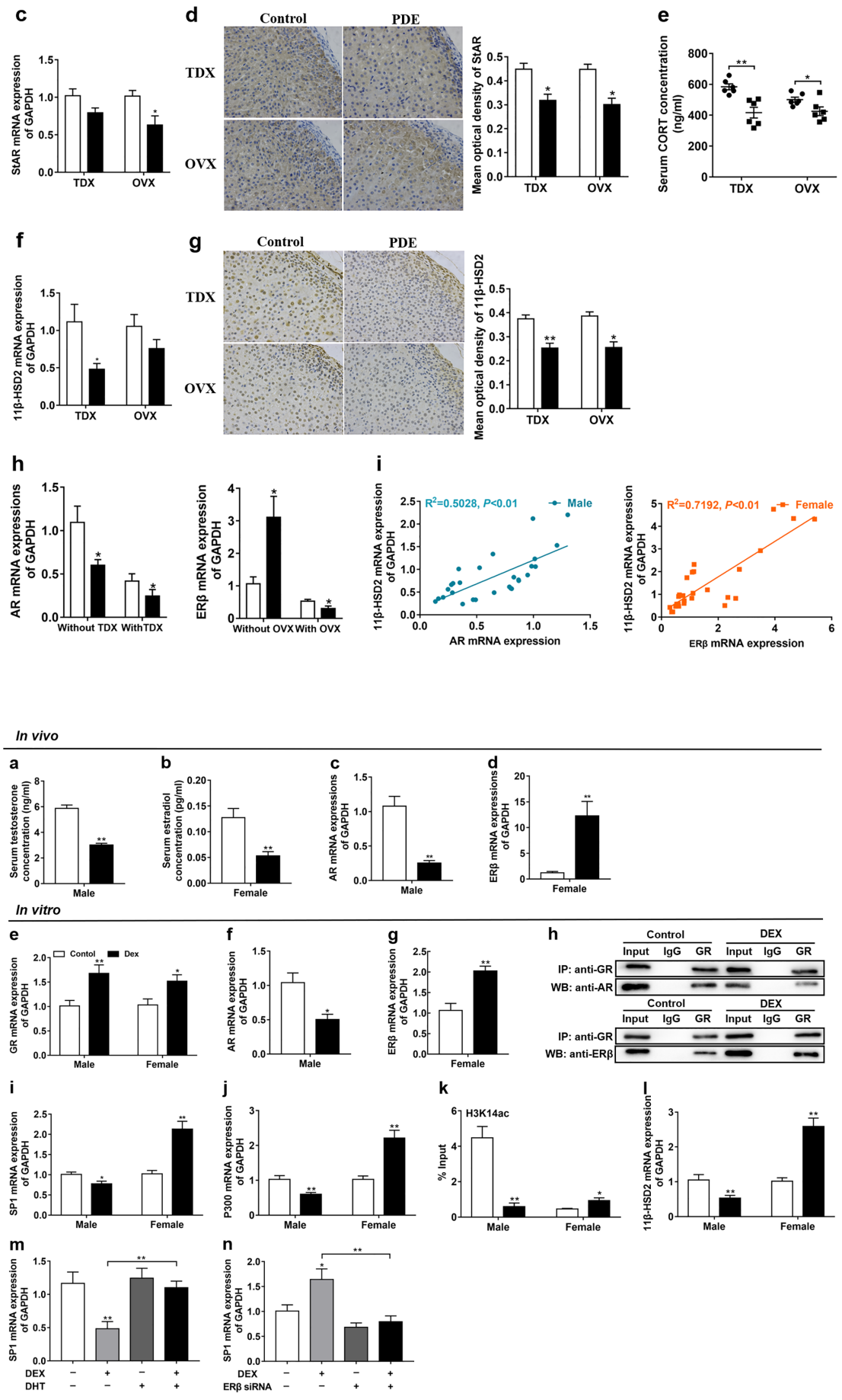

22 

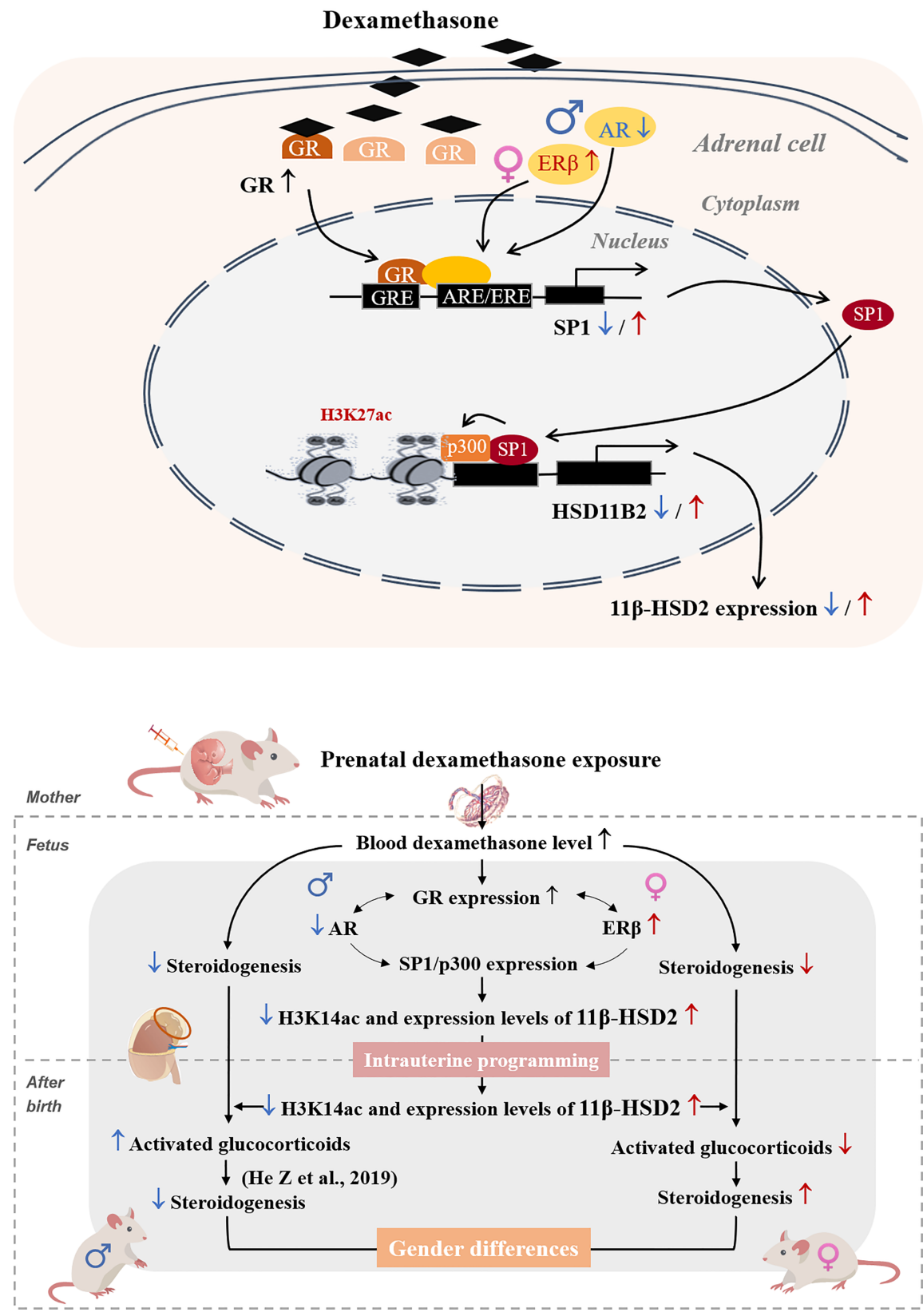